\title{
Prognostic impact of ICG-PDR in patients with hypoxic hepatitis
}

\author{
Thomas Horvatits ${ }^{1,2+}$, Nikolaus Kneidinger ${ }^{3 \dagger}$, Andreas Drolz ${ }^{1,2}$, Kevin Roedl ${ }^{1,2}$, Karoline Rutter ${ }^{1,2}$, Stefan Kluge ${ }^{2}$, \\ Michael Trauner ${ }^{1}$ and Valentin Fuhrmann ${ }^{1,2^{*}}$
}

\begin{abstract}
Background: Hepatic impairment is found in up to $20 \%$ in critically ill patients. Hypoxic/ischemic hepatitis (HH) is a diffuse hepatic damage associated with high morbidity and mortality. Indocyanine green plasma disappearance rate (ICG-PDR) is an effective tool assessing liver function in acute and chronic hepatic diseases. Aim of this study was to evaluate the prognostic impact of ICG-PDR in comparison to established parameters for risk stratification.
\end{abstract}

Methods: Patients with $\mathrm{HH}$ were included in this prospective observational study and compared to cirrhosis, acute liver failure (ALF) and patients without underlying liver disease. ICG-PDR, measured non-invasively by finger pulse densitometry, was assessed on admission and in patients with $\mathrm{HH}$ serially and results were compared between groups. Diagnostic test accuracy of ICG-PDR predicting 28-day mortality was analyzed by receiver operating characteristics (ROC).

Results: ICG-PDR on admission was significantly lower in patients with liver diseases than in patients without hepatic impairment (median $5.7 \% / \mathrm{min}$, IQR 3.8-7.9 vs. $20.7 \% / \mathrm{min}$, IQR 14.1-25.4\%/min; $p<0.001$ ). ICG-PDR predicted 28-day mortality independently of SOFA score and serum lactate in patients with underlying liver disease (HR 1.27, $95 \% \mathrm{Cl} 1.10-1.45, p<0.001)$. In patients with HH, ICG-PDR was identified as best predictor of 28-day mortality which performed significantly better than SOFA, lactate, INR and AST over course of time $(p<0.05)$. Best cut-off for identification of 28-day survivors was ICG-PDR $\geq 9.0 \% / \mathrm{min} 48 \mathrm{~h}$ after admission.

Conclusions: ICG-PDR is an independent predictor of mortality in patients with liver disease. Diagnostic test accuracy of ICG-PDR was superior to standard liver function parameters and established scoring systems in patients with $\mathrm{HH}$.

Keywords: Hypoxic hepatitis, Ischemic hepatitis, Indocyanine green plasma disappearance rate, ICG-PDR

\section{Background}

Hepatic impairment is a frequent finding in critically ill patients. Cholestatic liver dysfunction is found in up to $20 \%$, whereas hypoxic liver injury is found in up to $10 \%$ of patients admitted to medical intensive care units (ICU) $[1,2]$. Both, cholestatic as well as hypoxic liver dysfunction is associated with increased mortality [3-5]. Acute liver failure (ALF) is a life-threatening but rare disease,

\footnotetext{
*Correspondence: v.fuhrmann@uke.de

${ }^{\dagger}$ Horvatits Thomas and Kneidinger Nikolaus contributed equally to the work

${ }^{2}$ Department of Intensive Care Medicine, University Medical Center Hamburg-Eppendorf, Hamburg, Germany

Full list of author information is available at the end of the article
}

with an incidence of less than 10 cases/million persons [6]. Acute-on chronic liver failure, defined as acute decompensation of cirrhosis, is characterized by rapid progression and high mortality rates [7].

Hypoxic hepatitis (HH), also known as ischemic hepatitis or shock liver, is a diffuse hepatic damage characterized by centrilobular liver cell necrosis associated with high morbidity and mortality $[2,4,5]$. $\mathrm{HH}$ is defined as a sharp increase of aminotransferase levels in an acute setting of cardiac, septic or respiratory failure. $\mathrm{HH}$ is associated with high mortality rates up to more than $50 \%[2$, $5,8-10]$. Laboratory parameters as serum aspartate aminotransferase level (AST), international normalized ratio (INR) and arterial serum lactate have been described 
to be elevated in $\mathrm{HH}$ non-survivors [9]. However, there is still a lack of prognostic tool for risk stratification in patients with $\mathrm{HH}$.

The fluorescent medical dye indocyanine green (ICG) is taken up exclusively by hepatocytes and is secreted unchanged into the bile. As ICG is removed solely by the liver without entering enterohepatic circulation, it has been described as an effective quantitative liver function test $[11,12]$. Elimination of ICG represents both, hepatic blood flow as well as parenchymal function. ICG clearance has been described for assessing liver function in patients undergoing liver surgery (hepatic resection in patients with hepatocellular carcinoma $(\mathrm{HCC})$ ) as well as in donors and recipients of living donor liver transplantation (OLT) [13-15]. Furthermore, indocyanine green plasma disappearance rate (ICG-PDR) has been evaluated as prognostic marker in patients with advanced liver cirrhosis $[16,17]$. Recently, ICG clearance has been described as an effective tool for assessing portal hypertension in patients with compensated liver cirrhosis [18]. Additionally, ICG-PDR serves as a good predictor for mortality in critically ill patients [12, 19-21].

However, there is a lack of data regarding ICG clearance in critically ill patients with hepatic impairment. In particular, there are no data regarding ICG clearance in patients with $\mathrm{HH}$ available.

This study evaluates the prognostic impact of ICGPDR, in comparison to established parameters for risk stratification, in patients with $\mathrm{HH}$ and compares it to patients with ALF, decompensated liver cirrhosis or without underlying liver disease at the ICU.

\section{Patients and methods Patients}

In this observational study, impact of ICG-PDR was prospectively evaluated and compared to established parameters for risk stratification. Patients with $\mathrm{HH}$ were compared to patients with ALF or liver cirrhosis admitted to the ICU and critically ill patients suffering from severe shock but without liver disease. Exclusion criteria were age $<18$, pregnancy, HCC, previous OLT, presence of extracorporeal life support system, and presence of hyperthyroidism and iodine allergy according manufacturers product information. ICG-PDR was assessed in between therapy cycles in patients requiring renal replacement therapy (RRT).

Data collection was performed on daily basis. Sequential Organ Failure Assessment (SOFA) [22], Simplified Acute Physiology Score II (SAPSII) [23], Model of Endstage Liver Disease (MELD) [24] were calculated on ICU admission as well as 24 and $48 \mathrm{~h}$ thereafter. MELD score was calculated for patients with $\mathrm{HH}$ and controls as well. However, MELD score is routinely used mainly in patients with cirrhosis and ALF. Shock index (SI) was calculated as ratio of heart rate and systolic blood pressure as established representative of systemic hemodynamics [25]. Vasopressor support and dosage were recorded. 28-day mortality was prospectively assessed.

The study protocol was approved by the Ethics Committee of the Medical University of Vienna and performed according to the ethical principles of the revised Helsinki declaration. Patients' informed consent was obtained.

\section{Definitions}

Diagnosis of $\mathrm{HH}$ was made according the well-established criteria: (a) setting of cardiac, circulatory or respiratory failure, (b) dramatic but transient elevation in serum AST levels to at least 20-fold the upper limit of normal, (c) exclusion of other putative causes of liver cell necrosis (viral or drug induced hepatitis) [8]. Patients with $\mathrm{HH}$ accompanying cirrhosis were excluded from the study.

Liver cirrhosis was diagnosed by histological or by combination of clinical signs and laboratory findings or by typical radiologic signs in abdominal ultrasonography or computed tomography scan.

Acute liver failure was defined according to the international consensus as INR $>1.5$ and the presence of hepatic encephalopathy in patients without liver cirrhosis and duration of the disease (jaundice) of less than 26 weeks [26].

\section{Measurement of ICG-PDR}

For each measurement ICG ( $0.25 \mathrm{mg} / \mathrm{kg}$ bodyweight $)$ was injected intravenously via a central line followed by $5 \mathrm{ml}$ of normal saline, as described elsewhere [27]. Plasma disappearance rate of ICG was measured non-invasively by using a finger pulse densitometry system (LiMON, Pulsion Medical Systems SE, Munich, Germany) [28] (normal range of ICG-PDR is $18-25 \% / \mathrm{min}$ ). ICG-PDR was assessed in all patients on admission and in patients with HH serially on a daily basis until day 5 . ICG measurement was not possible due to poor peripheral perfusion as consequence of severe shock in two patients with $\mathrm{HH}$.

\section{Management}

Patients with septic or cardiogenic shock were treated according to standardized protocols [29, 30]. Intravenous fluid administration as well as vasopressor therapy was initiated in patients meeting shock criteria aiming to maintain a mean arterial blood pressure of $>65 \mathrm{mmHg}$. Early initiation of broadspectrum antibiotic treatment was performed according standardized protocols [29]. Antimicrobial therapy was adapted to culture results. RRT was performed in patients with renal failure and/ 
or metabolic acidosis. Patients with suspected acetaminophen-associated ALF received intravenous $N$-acetylcysteine [31].

\section{Data analysis and statistics}

Data were described as median and 25-75 \% interquartile range (IQR). Metric variables were compared using Mann-Whitney $U$ test and dichotomous variables were compared using Chi-square analysis. Correlation analysis was performed using Spearman's correlation. Cox regression proportional hazard analysis was performed to assess predictors of 28-day mortality. A forward stepwise procedure was used to identify most potent predictors.

The overall diagnostic test accuracy of ICG-PDR, SOFA, arterial serum lactate, AST levels and INR was assessed by receiver operating characteristics (ROC) expressed as their area under the curve (AUROC). We compared AUROCs at several time points using standard non-parametric methods. Estimates of diagnostic test accuracy (sensitivity, specificity) were calculated using standard methods. One-way repeated/multiple measured analysis of variance (ANOVA) was performed in patients with $\mathrm{HH}$ surviving until day 5. A Greenhouse-Geisser correction was used for sphericity. For data management and analyses, we used MS Excel 2008 for Mac, SPSS 21 for Mac (SPSS, Inc. Chicago, IL, USA), and Stata 12 for Mac (Stata Corp., College Station, TX, USA). All $p$ values reported are two sided and $p<0.05$ was considered significant.

\section{Results}

\section{Patients' characteristics}

A total of 97 patients were included in this observational study.

This cohort comprised 52 patients with $\mathrm{HH}, 35$ patients with cirrhosis admitted to the ICU because of acute decompensation and 10 patients with ALF.

Main reasons for occurrence of $\mathrm{HH}$ were cardiogenic $(n=32)$ and septic $n=20)$ shock. Only the first episode of $\mathrm{HH}$ that occurred during the stay at the ICU was assessed in this study.

Cause of ALF was acetaminophen intoxication $(n=3)$, non-acetaminophen drug induced $(n=2)$, or occurred as consequence of viral hepatitis $(n=2)$ and unknown reasons $(n=3)$.

The most common cause of cirrhosis was alcoholic liver disease $(n=18)$, followed by viral hepatitis $(n=12)$ and others $(n=5)$. The main ICU-admission diagnosis in patients with cirrhosis was sepsis/septic shock $(n=14)$ followed by coma hepaticum/hepatic encephalopathy grade 3-4 $(n=11)$ and hemorrhagic shock due to gastrointestinal bleeding $(n=10)$.
The control group included 22 critically ill patients. Main admission diagnoses were cardiogenic $(n=12)$ and septic $(n=10)$ shock without signs of severe acute liver injury, viral or drug induced hepatitis or cirrhosis. Detailed clinical characteristics of the patients are illustrated in Table 1.

SOFA score as well as SAPS II score on admission were significantly higher in patients with $\mathrm{HH}$ compared to cirrhosis and controls $(p<0.05)$. MELD score was significantly elevated in patients with $\mathrm{HH}$, cirrhosis and ALF in comparison to control patients $(p<0.001)$. Vasopressor support and hemodynamics were comparable between controls and patients with $\mathrm{HH}$ as shown in Table 1. ICGPDR did not correlate with SI, norepinephrine or dobutamine dose neither in the overall group of patients with underlying liver disease (spearman's $r=0.03, p=$ n.s.; $r=0.01, p=$ n.s.; $r=0.17, p=$ n.s.) nor in patients with $\mathrm{HH}$ (spearman's $r=0.09, p=$ n.s.; $r=-0.08, p=$ n.s.; $r=0.15, p=$ n.s.), respectively. Furthermore, ICG-PDR did neither correlate with CVP (central venous pressure) (spearman's $r=-0.1, p=$ n.s.), central venous oxygen saturation, (spearman's $r=0.02, p=$ n.s.) nor with $\mathrm{PaO}_{2} /$ $\mathrm{FiO}_{2}$ ratio (spearman's $r=-0.1, p=$ n.s.).

Aminotransferase levels on admission were significantly higher in patients with $\mathrm{HH}$ in comparison to patients with liver cirrhosis or patients without liver disease $(p<0.001)$. Furthermore, highest arterial serum lactate levels were found in patients with $\mathrm{HH}$ and ALF. Patients with $\mathrm{HH}$ showed significantly lower serum bilirubin levels than patients with cirrhosis and ALF $(p<0.001)$. ICG-PDR correlated with bilirubin in the overall cohort of patients with underlying liver disease as well as in patients with $\mathrm{HH}$ (spearman's $r=-0.47, p<0.001 ; r=-0.48, p<0.001$ ). Detailed laboratory data are illustrated in Table 2. Median ICU length of stay was 9 days (IQR 4-15 days), whereas median hospital length of stay was 13 days (IQR 6-27 days). One patient with ALF underwent OLT.

\section{ICG-PDR on admission and outcome in critically patients with liver diseases}

ICG-PDR on admission was significantly lower in patients with liver diseases $(\mathrm{HH}$, liver cirrhosis, ALF) than in the control group without hepatic impairment (median $5.7 \% / \mathrm{min}$, IQR $3.8-7.9$ vs. $20.7 \% / \mathrm{min}, \mathrm{IQR}$ $14.1-25.4 \% / \mathrm{min} ; p<0.001)$ as illustrated in Fig. 1.

There was no significant difference of ICG-PDR between $\mathrm{HH}$ and ALF. Detailed data of ICG-PDR is shown in Table 2.

ICG-PDR was significantly higher in 28-day survivors compared to non-survivors in the overall group of patients with liver disease (median $6.6 \% / \mathrm{min}$, IQR $4.8-$ $9.1 \% / \mathrm{min}$ vs. median $4.9 \% / \mathrm{min}$, IQR $3.4-6.6 \% / \mathrm{min}$; 
Table 1 Patient characteristics

\begin{tabular}{|c|c|c|c|c|c|}
\hline & Patients with liver disease & $\mathrm{HH}$ & ALF & Cirrhosis & Control \\
\hline \multicolumn{6}{|l|}{ Parameter } \\
\hline$n$ & 97 & 52 & 10 & 35 & 22 \\
\hline Male, $(\%)$ & $61(63)$ & $34(65)$ & $4(40)$ & $23(66)$ & $16(73)$ \\
\hline Age, years ${ }^{a}, b, e$ & $54(44-66)$ & $64(47-76)$ & $40(35-49)$ & $48(39-57)$ & $53(44-67)$ \\
\hline SOFA $A^{b, c}$ & $12(8-16)$ & $13(9-16.8)$ & $9(5.8-14)$ & $11(7-14)$ & $10.5(7-13)$ \\
\hline SAPS $\|^{a, b, c}$ & $57(38-71)$ & $60.5(43.3-78.5)$ & $26.5(15.8-56.5)$ & $51(35-67)$ & $50(36-59)$ \\
\hline MELD ${ }^{a, c}, d, e, f$ & $22(17-30)$ & $21.5(15-28.8)$ & $31(24.5-35.3)$ & $19(17-30)$ & $8(6-14.5)$ \\
\hline Mechanical ventilation, $(\%)^{\mathrm{e}, \mathrm{f}}$ & $75(77)$ & $42(81)$ & $7(70)$ & $26(74)$ & $21(96)$ \\
\hline Vasopressor, (\%) & $74(76)$ & $45(87)$ & $4(50)$ & $24(69)$ & $17(77)$ \\
\hline Mean arterial pressure, $\mathrm{mmHg}$ & $72(65.5-83.5)$ & $71.5(68.3-77)$ & $75.5(66.8-88.3)$ & $70(65-80)$ & $72(65.5-83.5)$ \\
\hline Heart rate, beat/min & $94(80-111.5)$ & $95(83-113.8)$ & $93.5(83.3-105)$ & $92(80-113)$ & $90(76.8-105)$ \\
\hline Renal replacement therapy, (\%) & $39(40)$ & $21(40)$ & $6(60)$ & $12(34)$ & $5(23)$ \\
\hline 28-day mortality, (\%) ${ }^{\mathrm{a}, \mathrm{d}}$ & $48(49)$ & $27(52)$ & $1(10)$ & $20(57)$ & $7(32)$ \\
\hline
\end{tabular}

Data are shown as median and IQR or as number and percentage

a $p<0.05 \mathrm{HH}$ vs. ALF

b $p<0.05 \mathrm{HH}$ vs. cirrhosis

c $p<0.05 \mathrm{HH}$ vs. control

d $p<0.05$ ALF vs. cirrhosis

e $p<0.05$ ALF vs. control

f $p<0.05$ cirrhosis vs. control

Table 2 Laboratory characteristics

\begin{tabular}{|c|c|c|c|c|c|}
\hline & Patients with liver disease & $\mathrm{HH}$ & ALF & Cirrhosis & Control \\
\hline \multicolumn{6}{|l|}{ Parameter } \\
\hline$n$ & 97 & 52 & 10 & 35 & 22 \\
\hline ICG-PDR, \%/min ${ }^{b, c, e, f}$ & $5.7(3.8-7.9)$ & $7(4.7-8.9)$ & $5.7(4.8-8.0)$ & $4.1(3.4-5.9)$ & $20.7(14.1-25.4)$ \\
\hline Bilirubin, mg/dl ${ }^{a, b}, c, e, f$ & $3.7(1.7-9.7)$ & $2.2(0.8-3.8)$ & $23.8(9.1-29.2)$ & $7.7(3.3-16.6)$ & $0.8(0.6-1.3)$ \\
\hline$I N R^{a, c, d, e, f}$ & $1.8(1.5-2.3)$ & $1.7(1.4-2.3)$ & $3.3(2.1-5.2)$ & $1.7(1.4-2.2)$ & $1.2(1.1-1.3)$ \\
\hline AST, U/l $\left.\right|^{b, c, d, e}$ & $1096(133-3798)$ & $2667(1267-5015)$ & $1815(216-5289)$ & $84(55-202)$ & $67(38-418)$ \\
\hline$A L T, U / I^{b, c, d, e}$ & $743(76-2119)$ & $1430(871-2417)$ & $2224(202-6173)$ & $43(24-93)$ & $54(21-176)$ \\
\hline Lactate, $\mathrm{mmol} / \mathrm{I}^{\mathrm{b}}$ & $3.5(2-6.2)$ & $4(2.3-9.3)$ & $4(2.3-5.3)$ & $2.5(1.8-4)$ & $3(1.8-3.6)$ \\
\hline Creatinine, $\mathrm{mg} / \mathrm{dl}^{\mathrm{a}, \mathrm{c}}$ & $1.5(1-2.4)$ & $1.7(1.3-2.3)$ & $0.9(0.7-1.5)$ & $1.2(0.8-3.1)$ & $1.1(0.8-2)$ \\
\hline
\end{tabular}

Data are shown as median and IQR

AST aspartate aminotransferase level, $A L T$ alanine aminotransferase level, INR international normalized ratio

a $p<0.05 \mathrm{HH}$ vs. ALF

${ }^{\mathrm{b}} p<0.05 \mathrm{HH}$ vs. cirrhosis

c $p<0.05 \mathrm{HH}$ vs. control

d $p<0.05$ ALF vs. cirrhosis

e $p<0.05$ ALF vs. control

f $p<0.05$ cirrhosis vs. control

$p<0.001)$ as well as in the group of patients with $\mathrm{HH}$ (median $8.6 \% / \mathrm{min}$, IQR $6.3-10.5 \% / \mathrm{min}$ vs. median 5.4 , IQR $3.5-7.2 \% / \mathrm{min}, p<0.001)$.

Univariate cox regression analysis detected a significant association of ICG-PDR, SOFA score and arterial serum lactate on admission with 28-day mortality (HR 1.31,
95 \% CI 1.15-1.50, $p<0.001$; HR 1.12, 95 \% CI $1.04-$ $1.19, p<0.001$; HR 1.12, 95 \% CI 1.07-1.18, $p<0.001$ ) in patients with liver disease. In contrast, there was no association of age, sex, aminotransferase levels, INR and bilirubin levels with 28-day mortality. ICG-PDR remained an independent predictor of 28-day mortality even after 


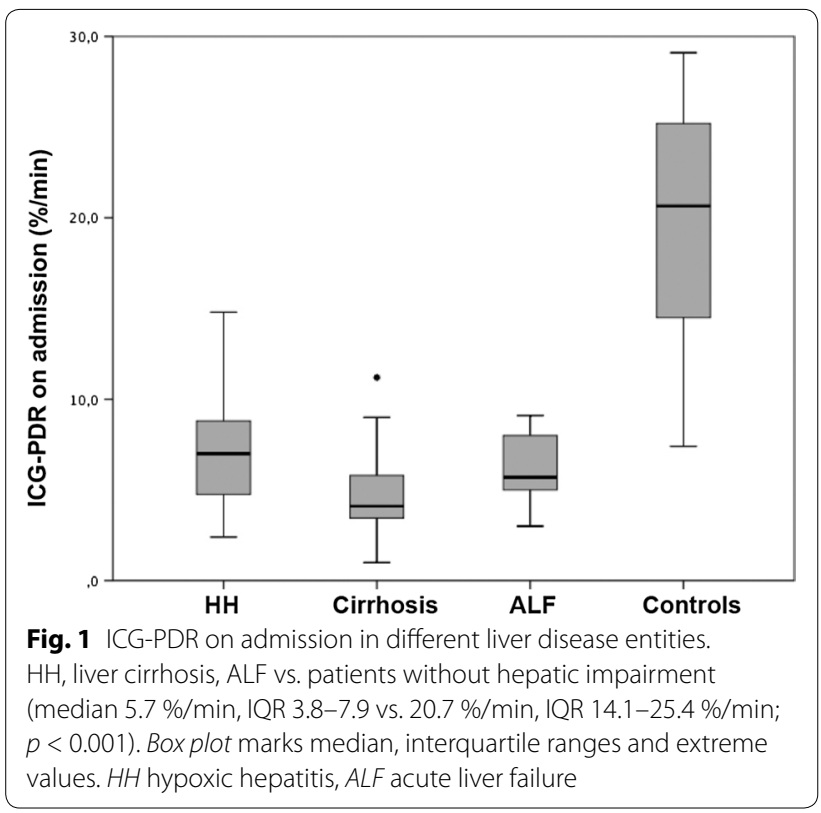

correction with SOFA score and arterial serum lactate in multivariate cox regression model (HR 1.27, $95 \% \mathrm{CI}$ $1.10-1.45, p<0.001)$. ROC analysis revealed that ICGPDR had the highest prediction of 28-day mortality, followed by arterial serum lactate and SOFA (Table 3).

\section{ICG-PDR and outcome in patients with hypoxic hepatitis}

ICG-PDR had highest AUROC of all parameters assessed on admission in regard to 28-day mortality as shown in Fig. 2. $48 \mathrm{~h}$ after admission, AUROC of ICG-PDR was significantly higher than AUROC of SOFA, arterial serum lactate, INR and AST ( $p<0.05$; see Table 4$)$. AUROC of $\Delta$-ICG as increase of ICG-PDR from admission to $48 \mathrm{~h}$ value was 0.85 .

Youden index showed best discrimination of 28-day survivors and non-survivors at a cut-off of ICG-PDR $\geq 7.8 \% / \mathrm{min}$ on admission (sensitivity of $68 \%$, specificity of $85 \%$ ) and $\geq 9.0 \% / \mathrm{min} 48 \mathrm{~h}$ after admission (sensitivity $79 \%$, specificity $100 \%)$.

Change of ICG-PDR over time in 30 patients with HH is illustrated in Fig. 3. One way repeated measured analysis of variance (ANOVA) showed a significant effect of ICG-PDR over time in 28-day survivors (GreenhouseGeisser corrected $F=22.4, d f=2.5, p<0.001)$ but not in 28-day non-survivors ( $p=$ n.s.).

\section{Discussion}

Hepatic impairment by means of cholestatic or hypoxic liver disease is of pivotal prognostic relevance in critically ill patients. In particular, $\mathrm{HH}$ seems to contribute to dramatically high mortality rates $[2,4,5,8,9]$. Beside
Table 3 ROC analysis of various parameters assessed on ICU admission for prediction of 28-day mortality in patients with underlying liver disease

\begin{tabular}{lll}
\hline Parameter & AUROC & $\mathbf{9 5} \% \mathbf{C l}$ \\
\hline ICG-PDR $^{\mathrm{a}}$ & 0.73 & $0.63-0.83$ \\
Lactate $^{\mathrm{a}}$ & 0.72 & $0.62-0.83$ \\
SOFA $^{\mathrm{b}}$ & 0.69 & $0.58-0.79$ \\
MELD & 0.65 & $0.54-0.76$ \\
INR & 0.62 & $0.51-0.73$ \\
Bilirubin & 0.54 & $0.43-0.66$ \\
AST & 0.54 & $0.42-0.66$ \\
\hline
\end{tabular}

AST aspartate aminotransferase level, INR international normalized ratio

a AUROC $p<0.05$ ICG-PDR, Lactate vs. Bilirubin, AST

b AUROC $p<0.05$ SOFA vs. AST
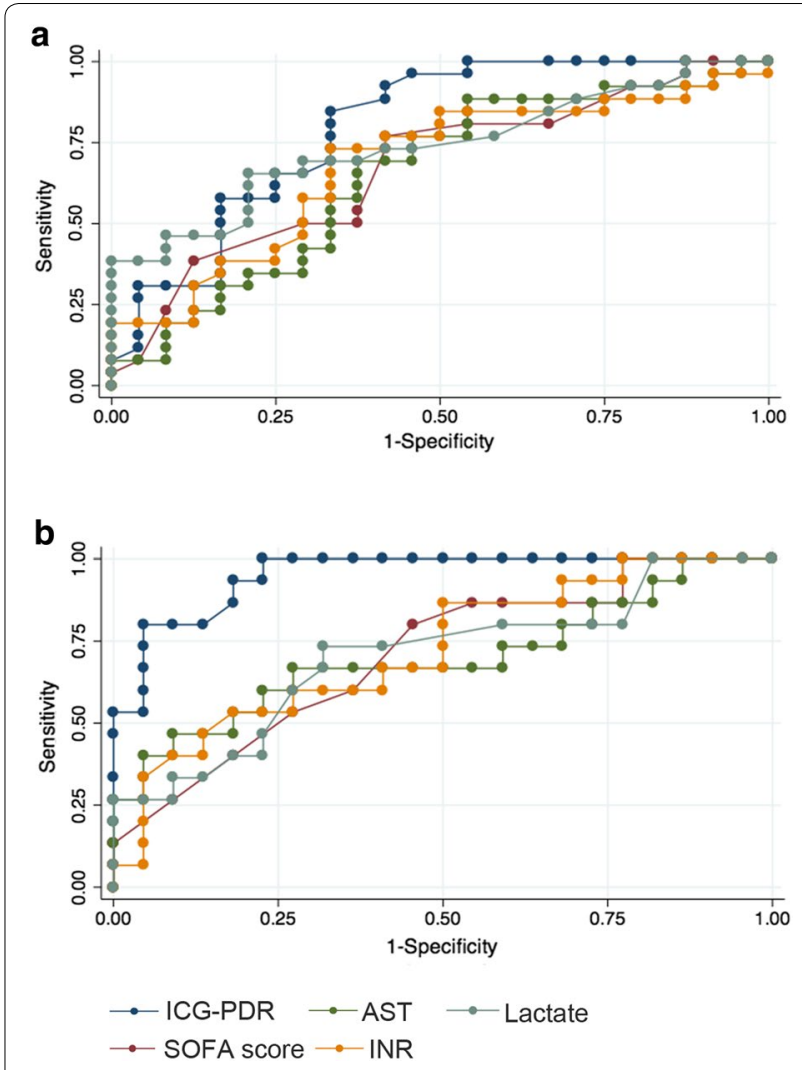

Fig. 2 ROC predicting 28-day mortality in patients with $\mathrm{HH}$ a on admission and $\mathbf{b} 48 \mathrm{~h}$ after admission. AST aspartate aminotransferase level, INR international normalized ratio

laboratory parameters, different quantitative liver function tests such as ICG clearance or maximal liver function capacity test (LiMAx) or tests for evaluation of hepatic blood flow (galactose clearance) aim to facilitate assessment of liver function in acute and chronic hepatic impairment $[4,32,33]$. ICG-PDR is reported 
Table 4 ROC analysis for predicting 28-day mortality in patients with $\mathrm{HH}$

\begin{tabular}{|c|c|c|}
\hline Parameter & AUROC & $95 \% \mathrm{Cl}$ \\
\hline BL-ICG-PDR & 0.81 & $0.68-0.93$ \\
\hline 24-ICG-PDR ${ }^{a}$ & 0.88 & $0.76-0.99$ \\
\hline $48-$ ICG-PDR $^{b}$ & 0.96 & $0.90-1.00$ \\
\hline BL-Lactate & 0.74 & $0.60-0.88$ \\
\hline 24-Lactate & 0.70 & $0.53-0.87$ \\
\hline 48-Lactate ${ }^{\text {b }}$ & 0.71 & $0.54-0.88$ \\
\hline BL-SOFA & 0.69 & $0.54-0.83$ \\
\hline 24-SOFA & 0.68 & $0.52-0.83$ \\
\hline $48-S O F A^{b}$ & 0.73 & $0.58-0.89$ \\
\hline BL-INR & 0.66 & $0.51-0.81$ \\
\hline $24-\mathrm{INR}^{\mathrm{a}}$ & 0.69 & $0.53-0.85$ \\
\hline 48-INR & 0.76 & $0.61-0.91$ \\
\hline BL-AST & 0.63 & $0.48-0.78$ \\
\hline 24-AST & 0.76 & $0.61-0.90$ \\
\hline $48-A S T^{b}$ & 0.72 & $0.54-0.89$ \\
\hline
\end{tabular}

AST aspartate aminotransferase level, INR international normalized ratio

a AUROC $p<0.05$ 24-ICG-PDR vs. 24-INR

${ }^{b}$ AUROC $p<0.05$ 48-ICG-PDR vs. 48-Lactate, 48-SOFA, 48-INR, 48-AST

as prognostic parameter in stable patients with cirrhosis and in critically ill patients without underlying liver disease [17-21]. The LiMAx test was described as prognostic marker in liver transplant candidates as well as in patients with sepsis-related liver failure [32, 33].
However, none of the quantitative liver function tests were evaluated in the setting of acute hypoxic liver injury. Therefore, this study evaluates the prognostic impact of ICG-PDR in critically ill patients with HH compared to other forms of acute and chronic liver failure.

We could demonstrate that the degree of reduced ICGPDR was comparable in patients with $\mathrm{HH}$ and patients with ALF. In contrast, control patients with similar severity of shock without evident hepatic impairment had significantly higher ICG-PDR. ICG-PDR levels in critically ill patients with cirrhosis were consistent with previous studies [17].

Furthermore, we could demonstrate a strong association of ICG-PDR with 28-day mortality in critically ill patients with liver disease. Best prediction of 28-day mortality was found in patients with $\mathrm{HH}$. Additionally, diagnostic test accuracy of ICG-PDR was increasing over time in patients with $\mathrm{HH} .48 \mathrm{~h}$ after admission ICG clearance performed significantly better than SOFA score, arterial serum lactate, INR or AST in prediction of 28-day mortality. A previous study identified INR as well as SOFA score as independent predictors for overall mortality in patients with $\mathrm{HH}$ [9]. Diagnostic test accuracy of ICG-PDR was superior compared to standard liver function parameters as well as SOFA score in our cohort. Furthermore, the dynamics of ICG-PDR over time demonstrated that ICG clearance did not increase over the course of time in patients that died within the first 28 days, whereas a constant increase was observed

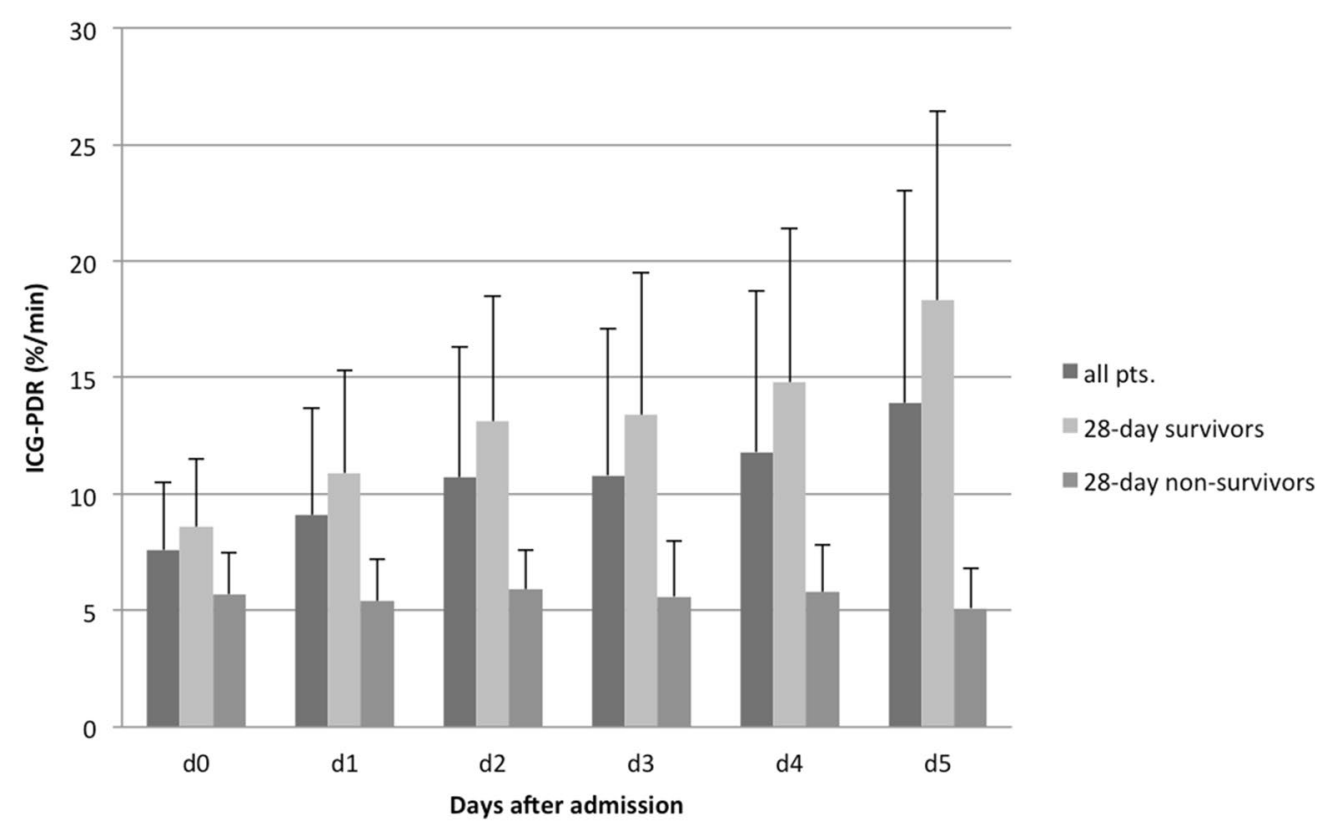

Fig. 3 Time course of ICG-PDR in patients with HH. ICG-PDR measurement on 4 consecutive days after admission in 30 patients with HH that survived until day 5. Repeated measures ANOVA showed a significant effect of time of ICG-PDR in 28-day survivors $(p<0.001)$ 
in survivors (see Fig. 3). Therefore, assessment of ICGPDR during the course of the disease seems to be of particular prognostic relevance. 28-day survivors could be identified using a cut-off ICG-PDR $\geq 9.0 \% / \mathrm{min} 48 \mathrm{~h}$ after admission with sensitivity of $77 \%$ and specificity $100 \%$.

ICG clearance is frequently used as global marker of hepatic function as it illustrates hepatic purification and also hepatic blood flow [11]. ICG-PDR did not correlate with SI, CVP, norepinephrine or dobutamine dose, respectively. Furthermore, the control group of our cohort with comparable etiology and severity of shock but without $\mathrm{HH}$ or any other cause of acute or chronic liver dysfunction had significantly higher ICG-PDR levels, indicating a strong association of ICG-PDR and hepatic function also in patients with severe shock. This is in accordance with another study that observed no association of alterations of cardiac output and a change in flow-dependent hepatic function with respect to ICG-PDR in critically ill patients without clinical manifestation of hepatic impairment [34].

There are some limitations of our study. First, the sample size of our study was rather small. However, this is the first prospective study investigating the prognostic impact of ICG-PDR in critically ill patients with life-threatening acute and chronic liver diseases. Second, this study was conducted at a medical ICU of a university hospital. Thus, conclusions drawn in this study may not be generalizable to patients treated in surgical or tertiary care hospital wards.

\section{Conclusions}

In summary, we could demonstrate that ICG-PDR is a strong and independent predictor of 28-day mortality in critically ill patients with underlying liver disease. Diagnostic test accuracy of ICG-PDR was superior to standard liver function parameters and well-established scoring systems in patients with $\mathrm{HH}$, which increased over course of time. ICG-PDR seems to be a bedside feasible non-invasive tool for early risk stratification in patients with $\mathrm{HH}$.

\footnotetext{
Abbreviations

$\mathrm{HH}$ : hypoxic hepatitis; ICU: intensive care unit; AST: aspartate aminotransferase level; INR: international normalized ratio; ICG: indocyanine green; ICG-PDR: indocyanine green plasma disappearance rate; ALF: acute liver failure; HCC: hepatocellular carcinoma; RRT: renal replacement therapy; OLT: liver transplantation; SOFA: Sequential Organ Failure Assessment; SAPSII: Simplified Acute Physiology Score II; MELD: model of end-stage liver disease; SI: shock index; HE: hepatic encephalopathy; IQR: interquartile range; ROC: receiver operating characteristics; AUROC: area under the ROC curve; ANOVA: analysis of variance; CVP: central venous pressure.
}

\section{Authors' contributions}

$H T$, KN: conception and design, performing measurements, drafting manuscript, statistical analysis and interpretation of data; DA, RK, RK: performing measurements, acquisition of data, drafting of manuscript; KS, TM: conception and design, critical revision of the manuscript for important intellectual content; FV: conception and design, performing measurements, drafting manuscript, statistical analysis and interpretation of data, critical revision of the manuscript. All authors read and approved the final manuscript.

\section{Author details}

${ }^{1}$ Division of Gastroenterology and Hepatology, Department of Internal Medicine 3, Medical University of Vienna, Vienna, Austria. ${ }^{2}$ Department of Intensive Care Medicine, University Medical Center Hamburg-Eppendorf, Hamburg, Germany. ${ }^{3}$ Department of Internal Medicine V, Comprehensive Pneumology Center (CPC-M), Member of the German Center for Lung Research (DZL), University of Munich, Munich, Germany.

\section{Acknowledgements}

No financial support has been received for conducting this study.

\section{Competing interests}

The authors declare that they have no competing interests.

Received: 25 June 2015 Accepted: 18 November 2015

Published online: 04 December 2015

\section{References}

1. Kramer L, Jordan B, DrumI W, Bauer P, Metnitz PG. Incidence and prognosis of early hepatic dysfunction in critically ill patients - a prospective multicenter study. Crit Care Med. 2007;35(4):1099-104. doi:10.1097/01. CCM.0000259462.97164.A0.

2. Horvatits T, Trauner M, Fuhrmann V. Hypoxic liver injury and cholestasis in critically ill patients. Curr Opin Crit Care. 2013;19(2):128-32. doi:10.1097/ MCC.0b013e32835ec9e6.

3. Jager B, Drolz A, Michl B, Schellongowski P, Bojic A, Nikfardjam M, et al. Jaundice increases the rate of complications and 1-year mortality in patients with hypoxic hepatitis. Hepatology. 2012;56(6):2297-304. doi:10.1002/hep.25896.

4. Henrion J, Schapira M, Luwaert R, Colin L, Delannoy A, Heller FR. Hypoxic hepatitis: clinical and hemodynamic study in 142 consecutive cases. Medicine (Baltimore). 2003;82(6):392-406. doi:10.1097/01 md.0000101573.54295.bd.

5. Fuhrmann V, Kneidinger N, Herkner H, Heinz G, Nikfardjam M, Bojic A, et al. Impact of hypoxic hepatitis on mortality in the intensive care unit. Intensive Care Med. 2011;37(8):1302-10. doi:10.1007/s00134-011-2248-7.

6. Bernal W, Wendon J. Acute liver failure. N Engl J Med. 2013;369(26):252534. doi:10.1056/NEJMra1208937.

7. Jalan R, Gines P, Olson JC, Mookerjee RP, Moreau R, Garcia-Tsao G, et al. Acute-on chronic liver failure. J Hepatol. 2012;57(6):1336-48. doi:10.1016/j.jhep.2012.06.026

8. Henrion J, Minette P, Colin L, Schapira M, Delannoy A, Heller FR. Hypoxic hepatitis caused by acute exacerbation of chronic respiratory failure: a case-controlled, hemodynamic study of 17 consecutive cases. Hepatology. 1999;29(2):427-33. doi:10.1002/hep.510290202.

9. Fuhrmann V, Kneidinger N, Herkner H, Heinz G, Nikfardjam M, Bojic A, et al. Hypoxic hepatitis: underlying conditions and risk factors for mortality in critically ill patients. Intensive Care Med. 2009;35(8):1397-405. doi:10.1007/s00134-009-1508-2.

10. Drolz A, Horvatits T, Michl B, Roedl K, Schellongowski P, Holzinger U, et al. Statin therapy is associated with reduced incidence of hypoxic hepatitis in critically ill patients. J Hepatol. 2014;60(6):1187-93. doi:10.1016/j. jhep.2014.01.019.

11. Cherrick GR, Stein SW, Leevy CM, Davidson CS. Indocyanine green: observations on its physical properties, plasma decay, and hepatic extraction. J Clin Invest. 1960;39:592-600. doi:10.1172/JCl104072.

12. Vos JJ, Wietasch JK, Absalom AR, Hendriks HG, Scheeren TW. Green light for liver function monitoring using indocyanine green? An overview of current clinical applications. Anaesthesia. 2014;69(12):1364-76. doi:10.1111/anae.12755.

13. Yokoyama Y, Nishio H, Ebata T, Igami T, Sugawara G, Nagino M. Value of indocyanine green clearance of the future liver remnant in predicting outcome after resection for biliary cancer. Br J Surg. 2010;97(8):1260-8. doi:10.1002/bjs.7084.

14. Poon RT, Fan ST. Hepatectomy for hepatocellular carcinoma: patient selection and postoperative outcome. Liver Transpl. 2004;10(2 Suppl 1):S39-45. doi:10.1002/lt.20040. 
15. Jochum C, Beste M, Penndorf V, Farahani MS, Testa G, Nadalin S, et al. Quantitative liver function tests in donors and recipients of living donor liver transplantation. Liver Transpl. 2006;12(4):544-9. doi:10.1002/It.20627.

16. Zipprich A, Kuss O, Rogowski S, Kleber G, Lotterer E, Seufferlein T, et al. Incorporating indocyanin green clearance into the Model for End Stage Liver Disease (MELD-ICG) improves prognostic accuracy in intermediate to advanced cirrhosis. Gut. 2010;59(7):963-8. doi:10.1136/ gut.2010.208595.

17. Stauber RE, Wagner D, Stadlbauer V, Palma S, Gurakuqi G, Kniepeiss $D$, et al. Evaluation of indocyanine green clearance and model for end-stage liver disease for estimation of short-term prognosis in decompensated cirrhosis. Liver Int. 2009;29(10):1516-20. doi:10.1111/j.1478-3231.2009.02104.x.

18. Lisotti A, Azzaroli F, Buonfiglioli F, Montagnani M, Cecinato P, Turco L, et al. Indocyanine green retention test as a noninvasive marker of portal hypertension and esophageal varices in compensated liver cirrhosis. Hepatology. 2014;59(2):643-50. doi:10.1002/hep.26700.

19. Sakka SG, Reinhart K, Meier-Hellmann A. Prognostic value of the indocyanine green plasma disappearance rate in critically ill patients. Chest. 2002;122(5):1715-20.

20. Zoller B, Spanaus K, Gerster R, Fasshauer M, Stehberger PA, Klinzing S, et al. ICG-liver test versus new biomarkers as prognostic markers for prolonged length of stay in critically ill patients-a prospective study of accuracy for prediction of length of stay in the ICU. Ann Intensive Care. 2014;4:19. doi:10.1186/s13613-014-0019-7.

21. Kortgen A, Paxian M, Werth M, Recknagel P, Rauchfuss F, Lupp A, et al. Prospective assessment of hepatic function and mechanisms of dysfunction in the critically ill. Shock. 2009;32(4):358-65. doi:10.1097/ SHK.0b013e31819d8204.

22. Vincent JL, Moreno R, Takala J, Willatts S, De Mendonca A, Bruining $\mathrm{H}$, et al. The SOFA (Sepsis-related Organ Failure Assessment) score to describe organ dysfunction/failure. On behalf of the Working Group on Sepsis-Related Problems of the European Society of Intensive Care Medicine. Intensive Care Med. 1996;22(7):707-10.

23. Le Gall JR, Lemeshow S, Saulnier F. A new Simplified Acute Physiology Score (SAPS II) based on a European/North American multicenter study. JAMA. 1993;270(24):2957-63.

24. Kamath PS, Wiesner RH, Malinchoc M, Kremers W, Therneau TM, Kosberg $\mathrm{CL}$, et al. A model to predict survival in patients with end-stage liver disease. Hepatology. 2001;33(2):464-70. doi:10.1053/jhep.2001.22172.
25. Mutschler M, Nienaber U, Munzberg M, Wolfl C, Schoechl H, Paffrath T, et al. The Shock Index revisited - a fast guide to transfusion requirement? A retrospective analysis on 21,853 patients derived from the TraumaRegister DGU. Crit Care. 2013;17(4):R172. doi:10.1186/cc12851.

26. Stravitz RT, Kramer AH, Davern T, Shaikh AO, Caldwell SH, Mehta RL, et al. Intensive care of patients with acute liver failure: recommendations of the U.S. Acute Liver Failure Study Group. Crit Care Med. 2007;35(11):2498508. doi:10.1097/01.CCM.0000287592.94554.5F.

27. Sakka SG, Koeck H, Meier-Hellmann A. Measurement of indocyanine green plasma disappearance rate by two different dosages. Intensive Care Med. 2004;30(3):506-9. doi:10.1007/s00134-003-2091-6.

28. Sakka SG, Reinhart K, Meier-Hellmann A. Comparison of invasive and noninvasive measurements of indocyanine green plasma disappearance rate in critically ill patients with mechanical ventilation and stable hemodynamics. Intensive Care Med. 2000;26(10):1553-6.

29. Dellinger RP, Levy MM, Rhodes A, Annane D, Gerlach H, Opal SM, et al. Surviving Sepsis Campaign: international guidelines for management of severe sepsis and septic shock, 2012. Intensive Care Med. 2013;39(2):165228. doi:10.1007/s00134-012-2769-8.

30. Werdan K, Russ M, Buerke M, Delle-Karth G, Geppert A, Schondube FA Cardiogenic shock due to myocardial infarction: diagnosis, monitoring and treatment: a German-Austrian S3 Guideline. Dtsch Arztebl Int. 2012;109(19):343-51. doi:10.3238/arztebl.2012.0343.

31. Lee WM, Hynan LS, Rossaro L, Fontana RJ, Stravitz RT, Larson AM et al. Intravenous $\mathrm{N}$-acetylcysteine improves transplant-free survival in early stage non-acetaminophen acute liver failure. Gastroenterology. 2009;137(3):856-64, 864 e851. doi:10.1053/j.gastro.2009.06.006.

32. Jara M, Malinowski M, Luttgert K, Schott E, Neuhaus P, Stockmann M. Prognostic value of enzymatic liver function for the estimation of shortterm survival of liver transplant candidates: a prospective study with the LiMAx test. Transpl Int. 2015;28(1):52-8. doi:10.1111/tri.12441.

33. Kaffarnik MF, Lock JF, Vetter H, Ahmadi N, Lojewski C, Malinowski M, et al. Early diagnosis of sepsis-related hepatic dysfunction and its prognostic impact on survival: a prospective study with the LiMAx test. Crit Care. 2013;17(5):R259. doi:10.1186/cc13089.

34. Sakka SG, Hofmann D, Thuemer O, Schelenz C, van Hout N. Increasing cardiac output by epinephrine after cardiac surgery: effects on indocyanine green plasma disappearance rate and splanchnic microcirculation. J Cardiothorac Vasc Anesth. 2007;21(3):351-6. doi:10.1053/j. jvca.2006.02.031.

\section{Submit your manuscript to a SpringerOpen ${ }^{\odot}$ journal and benefit from:}

- Convenient online submission

- Rigorous peer review

- Immediate publication on acceptance

- Open access: articles freely available online

- High visibility within the field

- Retaining the copyright to your article

Submit your next manuscript at $>$ springeropen.com 